\title{
Towards an Ontology of Information and Succeeding Fundamentals in Computing Science
}

\author{
Gerhard Luhn
}

gerhard.luhn@tu-dresden.de, Department of Computer Science, Technical University Dresden, Dresden, Germany.

\begin{abstract}
A common usage of the concept of information requires a unique definition of it. The text expands on a proposal for an ontology of information, which will be grounded in physics. Shannon's communication theory does not conceptualize any physical variable. It will be shown that by doing so the gap between syntax and semantics can be closed by introducing the universal category of triadic information. Any informational scenario is given by the trias of a) a sender, and of b) the transformation, which happens to c) the receiver. The concept of information is taken in a broader sense, and is based on physical fundaments. The gravitational force which is exploited to a physical body holds in the same sense well defined information as a spontaneous appearance of a new, algorithmically underivable structure or event: the world gets 'completed' within a continuous informational process. Any spontaneous process will always happen in order to increase the entropy of the world. That is, such fundamental information, which causes this completion process, is given within our universe. For those reasons we have to state, that information causes any causal process, rather than 'is' a causal process. All living species are grounded on an information-receiving, heteronomous deep structure, which includes as well the message which corresponds to further autonomy and freedom (the completion theorem). To summarize, we are enabled to create and to enter into the so called information society by ontological evidence. Based on such foundations, an adequate concept for computer science will be shortly introduced.
\end{abstract}

Keywords: universality of information, triadic information concept, identity based information transformation (iBIT)

Acknowledgement: This text is based on a speech which I gave at the "Information Theory and Practice" International Conference in Duino (Castle), Italy, Oct. 19th-21st 2007. I would like to thank all participants at this conference for their contributions and personal feedback. Special thanks to Hans Graßmann, Rafael Capurro and Koichiro Matsuno. I thank Johann Harter and Klaus Kabitzsch for personal and organizational support.

"The most incomprehensible thing about the world is that it is at all comprehensible." Albert Einstein

The forms of music and dances, the forms of whirls and galaxies, the forms of poems and of artworks, the forms of biological species, and also the forms of our thinking deliver a kind of information about something. This 'something' can be an object of the world, of our thinking or of both (i.e. artwork). We use the term 'form' in order to express some fundamental concepts of something. The interactions of such formations create specific instances of forms. We call this process information'. Rafael Capurro summarizes: "Information = instantiation of form" (Capurro, 2007, p. 283)

This concept holds two distinct movements. The first movement ('genitivus subjectivus') explains the process how matter gets formed. The second movement ('genitivus objectivus') deals with the epistemological dimension of information.

\section{Towards an Ontology of Information}

Information science is nowadays attempting to overcome the boundaries of an information concept, which has been based on statistics of messages between communicating systems. The information 
scientist Peter Rechenberg summarizes the actual debate. ${ }^{1}$ He states that information is the meaning/the content of a message, and such content cannot be measured. After Rechenberg, there seems to be a categorical gap between syntax and semantics.

Rechenberg is pointing to the fact, that this confusion "has generated damaging effects to this very day" (Rechenberg, 2003). Many contributions have been made to the concept of information within computing science. But there is one outcome: there has been little or even no impact on the design of computing systems. For this reasons an architectural proposal for new computing systems will be given; the structural identity to quantum computing will be shown. This architecture will be mapped into actual research projects. Those projects are part of the so-called "Cool Silicon" initiative of Saxony, and are conducted in the Department of Computer Science at Technical University Dresden.

\section{Fundamentals of a Triadic Information Concept}

What has physics to do with the concept of information?

Shannon's communication theory, and also Chaitin's algorithmic information theory do not conceptualize any physical variable. The physicist Hans Graßmann proposes a physical concept of digital messages (Graßmann, 2007).

Any digital message consists of a digital vector, for example $(0,1,1,0,0,1,0,1,1)$. Any communication of messages is defined by $b=\mathrm{T}(a)$; a: Input message, $b$ : output message, T: Transformation. T may be a computer: if we present him an input pattern, may be a video screen which contains a photo of you, the Transformation $\mathrm{T}$ could identify this photo and deliver your name as an output. The same holds true for the inverse direction: if we present your name to the computer, he might retrieve your photo out of his storage.

If it is true: $b=\mathrm{T}(a)$ and $a=\mathrm{T}^{-1}(b)$, then we have shown identity. The messages $a$ and $b$ are holding the same information. This information is given by the transformation $\mathrm{T}$.

What about the relation of digital and physical messages? Let us first recall the general concept of informational scenarios, which is given by the form

(1) $b=\mathrm{T}(a)$

We know different kinds of (physical) laws, but all are keeping the same form. Any (physical) law describes the fact that a specific conceptual entity (for example the force between two masses) is given by a mathematical combination of other independent entities (i.e. the weight and the distance of two masses). For this reason we may accept the form of (1) as a common structure of any physical law. $b$ might represent the magnitude of the gravitational force between two masses (typically described by the letter ' $F$ '), and $\mathrm{T}(\mathrm{a})$ represents the transformation which needs to be executed (in our example $T(a)=G^{*} m_{1}{ }^{*} m_{2} / r^{2} ; G$ : gravitational constant, $m_{1}$ : mass $1, m_{2}$ : mass 2 , r: distance between $\mathrm{m}_{1}$ and $\mathrm{m}_{2}$ ).

Our universe (as far as we know) exists of a limited and countable amount of energy. That is, any portion of energy can be counted by the smallest portion of energy, the Planck constant. For this reason we can count the masses $m_{1}$ and $m_{2}$. Within mathematics, such counted numbers are treated as natural numbers, and natural numbers are a fundamental category within our universe (Wilholt, 2004). Natural numbers and digital numbers are forming an injective relationship. For this reason we see that any quantitatively given physical entity can be translated into a digital value, without losing any information.

Within our example, the physical force between the two masses $F$ is caused by the two masses and will cause further transformations of the system. The input message (a) is given by the quantitative (may be digitalized) system description. Each input message holds an isomorphism to the output message. The output message is given by another quantitatively value. We see that the broader concept of 'message' and 'information' can be used even for the fundaments of physics.

\footnotetext{
${ }^{1}$ Luciano Floridi provides a semantic conception of Information, which enables a coherent usage of this 'notoriously polymorphic phenomenon' (Floridi, 2008). But his ontological conception ('there can be no information without data representation') has to be inverted: any data representation is caused by information.
} 
Physical laws are holding fundamental information. Of course one would argue that within the classical interpretation of information, such information is given by the reduction of uncertainty, which is given to us by knowledge of such physical laws. But where does the underlying, at least somehow certain process of information comes from, which reconstructs external information within the structure of matter and of living species? - Such information does certainly not come from uncertainty. This would be a contradictio in adjectio. The shapes of matter, of rocks, of living species are themselves mirroring the physical laws of the environment. The fins of fishes, the wings of birds are holding perfect fluid and aero dynamical shapes. For this reason I am arguing for the fundamental information of any physical law. Such kind of information gets not displayed on a semantic level of uncertainty, but enables any semantic reasoning. We have to give evidence to the fact that such fundamental information exists in a very certain manner.

This approach is inspired by Immanuel Kant's critique of judgment. He showed a very interesting path how knowledge about natural laws may appear within our mind, and includes already an idea about the 'unknown completion processes' of the world:

These laws, being empirical, may be contingent as far as the light of our understanding goes, but still, if they are to be called laws (as the concept of a nature requires), they must be regarded as necessary on a principle, unknown though it be to us, of the unity of the manifold. The reflective judgement which is compelled to ascend from the particular in nature to the universal stands, therefore, in need of a principle. This principle it cannot borrow from experience, because what it has to do is to establish just the unity of all empirical principles under higher, though likewise empirical, principles, and thence the possibility of the systematic subordination of higher and lower (Kant, 1790 [Emphasis GL]).

Nowadays the physicist and philosopher Carl Friedrich von Weizsäcker interpreted "information as form" with reference to Aristotle (Weizsäcker, 1980). This is another inspiring and modern approach, but Weizsäcker sticks still with the subsumption, that any informational process is guided and only guided - by physical laws. Kant's "empirical principles" and Weizsäcker's "information as form" are given to us by fundamental information. Such fundamental information offers a synthesis of the concepts of causal determinism and spontaneous causality.

The physicist Richard Feynman has pointed to a next fact. He is arguing for the fundamental status of quantum mechanics. He is arguing that the entire universe can be described by base vectors. The required equations are holding the same form as (1) and are creating a linear vector space (Feynman, 2005). What he is intending to say is that all natural phenomena are holding the form of a linear space. Any nonlinear system behavior appears on the macroscopic physical level. See the theory of weather, which is based on a deterministic multipart system. Weather reports are becoming of better and better quality by transforming the required equations into strictly linear computer programs. Biology is successfully introducing the concept of bioinformatics. Cells are seen as cognitive entities, which produce outputs from within a linear computation perspective (Shapiro, 2006, and Bauer, 2008). Cognitive Science relies explicitly on the concept of a linear vector space in order to conceptualize any cognitive behavior (Chruchland, 1992 and Haugland, 1997). Kant's epistemology - although not explicitly mentioned - refers to the concept of a linear vector space within his interpretation of space and time. If nature is fundamentally based on the concept of vector space, then such structure should be identifiable within epistemological structures. Such proof has been made by empirical epistemology (Vollmer, 1985).

Spontaneous processes like the sudden emission of photons by an atom do not show a causal behavior. But those processes do not violate the basic physical laws, i.e. the laws of conservation. Based on such spontaneous processes, new structures may be assembled. The world gets completed by such activities, and this is the basic concept what needs to be considered. There is further information existing, which causes any spontaneous behavior. Any spontaneous behavior will always increase the entropy of the world. This is the reason why it is happening. The cause of any spontaneous behavior lies not outside our universe. For this reason we can argue that such information exists in a fundamental way and permits a continuous completion process. 


\section{THESIS 1:}

Fundamental Form of Information: Information holds a Triadic Structure.

(a) The wholeness of each system is given by the transformation(s) $T$ the system may rule. 'Information' is a fundamental category, which holds the evidence of a fundamental law and which gives the possible transformations of any system by following triadic structure: $b=T(a)$; where $b$ : target system (vector), a: source system (vector), $T$ : transformation ( $T$ is representing the unchanging structure of the system). While the dyadic concept of information is focusing on the exchange of elements between systems, the triadic approach declares and expands on the overall scenario.

(b) The concept of $T$ includes the creation of new T's in situations, where existing T's do not deliver an adequate solution: new structures $T$ will appear and new kinds of systems will populate the scene (Annila and Kuismanen, 2009). Especially all kinds of living species (as information receivers) are deeply grounded on this information-receiving, heteronomous deep structure. Certain autonomy is required to increase the amount of possible system states from within an overall systems perspective.

(c) Any material instantiation of a system (particle, atom, molecule or a lager system) dissipates energy; the amount of this energy equals the binding energy of the elements of the system. This process is to be described as a linear transformation $b=T(a) . T$ is the information which causes the creation of $b$ out of a. This model includes sender, receiver and transformation rules. That is, we are changing from Shannon's dyadic communication scenario to a triadic information concept.

This approach would still not give much advantage, if we take $T$ as the notion of existing physical laws. The difference is that we assume huge amounts of situations within the world, which cannot be described by only looking at singular physical laws. The situation is much more subtle. Systems interweave and build different kinds of hierarchies. Given all the kinds of interactions, systems are ever completing themselves and the universe. What is important for us and holds the notion of the 'newness' within any information comes out of this ever-completing process, which happens in nature and in our mind. This includes also blocking situations. Further development of a system gets blocked, if the existing sources of spontaneous events will be decoupled from the structural core of the system. The core of any information concept has to incorporate an approach for the 'newness' within our world. If the development of systems is getting blocked then they stay at the level of Newton's mechanics and classical materialism. Such active information-completionprocess is an intrinsic fundament of our world.

Basil Hiley and Paavo Pylkkänen are proposing a concept of 'active information' (Hiley \& Pylkkänen, 2005), which roots back to ideas of David Bohm (Bohm, 1990). Bohm's interpretation of quantum physics foresees a quantum potential, which actively holds information about its environment and which 'guides' the path of particles by causally influencing the wave function of those particles. Bohm, Hiley and Pylkkänen conceptualize the mind as a nonlocal organized field, rather than a locally organized network. A main and very interesting proposal is to conceptualize the mind as a nonlocal field. Classical approaches in neuroscience and psychology see the functionality of the mind from within a local perspective. But those approaches are trying hard to deliver coherent solutions for the concept of knowledge. Never the less, neuroscience is agreeing on a 'system-asa-whole-perspective' and introduces adequate concepts like 'unmonitored learning' and 'adaptive, self-organizing systems' (Kohonen, 2001).

Knowledge is rather organized in analogy (or is a) physical field (a nonlocal 'Gestalt'), rather than a complicate semantic net. Within a nonlocal field, such potentials in terms of possible modifications of the system are linearly superposed and represent an entire set of possible solutions. This includes the development of the movement of the body in a given situation as well as the activation of further thinking processes in more unclear situations. Physicists like Roger Penrose are declaring, that Nature "identifies" the solution which gets realized by applying the principle of minimal energy (Penrose, 1989): each atom seems to sense what a number of other atoms are doing as they fall into place in concert. The final solution gets selected according the minimal amount of 
energy what is required in order to realize that solution. Such nonlocal, nonalgorithmic causal processes are given when a new macrostate gets created which is based on nonlocal rather than on local rules. Within the physical world the meaning of Kurt Gödel's incompleteness statement is changing towards a "Completion theorem": whenever no local solution is given to resolve a certain situation, a new transformation (algebra) gets created, which cannot be derived out of the existing transformations. Within such an overall sense, information causes any causal processes, and including any spontaneous process.

\section{Elements, Systems, Symbols: Structures of Fundamental Information Processing}

If two systems are exchanging conserved quantities, we call this a causal process. If a system gets perpetuated by an element (or a small system), we call this element/small system a signal. The "bigger" system ("bigger" in terms of mass / energy) changes its macrostate. The smaller system can be reflected (but may transfer an amount of energy), absorbed or break into smaller units. A certain system may send out an element (based on internal events). With this concept we can define any communication scenario between systems. An output message $b$ can become input message to another system. Typically, causal processes are described as local processes.

Within classical mechanics and also within quantum mechanics, we find an isomorphic relationship between interacting systems. The resolution of the equations are fully reversible and do not depend on a direction of time. This holds not true anymore for any spontaneous process (like the burst of a radioactive atom). Spontaneous processes deliver a homomorphism between the system(s) in state a (represented by vector $a$ ) and the system(s) in state $\mathrm{b}$ (represented by vector $b$ ). A homomorphism is a structure-preserving map between two vectors (within maths: between two algebraic structures). An isomorphism maps exactly one element of vector a to one element of vector $b$. A homomorphism maps one element of vector $a$ to one or more elements of vector $b$. Both scenarios are reversible transformations. But within physics, due to the second law of thermodynamics and due to statistics, homomorphic mappings are holding the character of irreversible transformations.

Crystals are symbolizing their own growth conditions. Macrophysical parameter (temperature, pressure, humidity etc.) 'control' and modulate the microphysical arrangements and are precursors for such symbols. Any living specie is stabilizing its structure by transforming kinds of matter of higher order ('nurture') into matter of lower order. The transforming process of such nurture releases energy, which gets transformed by the system into further activities. The basic force behind this behavior is the $2^{\text {nd }}$ law of thermodynamics: the outcome of this activity is a system which instantiates a higher amount of possible system states. Any kind of learning will happen spontaneously, because this process will increase the entropy of the world. Let us think about Darwin's finches, which lost their well known kinds of nurture. New kinds of cognitive structure (of symbols) are getting instantiated from within a spontaneous perspective.

\section{THESIS 2:}

Triadic Information is Causing the Meaning of any Symbol: Information holds a Universal State and Completes the World.

a) For living species, the internal representation of a symbol stays always in correlation with the overall triadic structure and holds the evidence of a physical / biological law. Such triadic information is causing the meaning of any symbol in a very fundamental manner. Now the entire, overall scenario of this fundamental information-completion-process becomes visible. If such a symbol becomes created and gets active, then a new fundamental ordering structure of the world becomes true. This process is not based on the material exchange of signals, but causes any signal to carry the kind of information which reflects this very new ordering structure. For this reason we see that it does not cost any time in order to 'communicate' such kinds of new ordering structures. Such ordering structures are getting instantiated in a universal, timeless manner. 
b) Information is not an entity similar to energy or matter, like Norbert Wiener stated (Wiener, 1948). But he was on the right path: Information holds the same status as what we call a physical law, or a biological law. What is similar to the theory of matter and energy (which is physics) is a theory of messages (Capurro, 2010) (Shannon's approach makes part of it).

c) Information is fundamental; it causes (from within a local and an overall, nonlocal perspective) the process of enfolding of the world. Information causes the forms of matter, and the forms of living. For living species, such forms are known to them as symbols and are holding an active state. It causes living systems to continuously increase the amount of possible system states (for any system as well as for the overall system). We may not be able to predict algorithmically the timestamp and the outcome of spontaneous events. But we do know that any spontaneous event will always increase the entropy of the world. That is the reason why it is happening. Though information holds a universal state, and completes the world from within an overall perspective.

And because this process is based on the non-algorithmic completion process of the world, it may and will create what we call mistakes, errors and blocking situations. A new structure may not extend, but hinder already existing transformation schemes of the living specie. 'Blocking situation' may occur, if the overall system structure gets fixed in a manner, that its existing (algorithmic) capabilities will be decreased, instead of increased. Any non-algorithmic extension of a system cannot be validated upfront, simply because there is no algorithm available. As a result, the overall structure and organization of the system may become 'blocked'. Please not, that there is a significant probability to this effect, which is fundamentally grounded to the non-algorithmic structure of the creation of a new structure. This creation process risks "errors" and "malfunctions", because this new structure may only have been approximately verified from within the deterministic parts of system behavior. But on the other hand, biology is just now discovering an immense capability of simulating changes of genetic items within an anticipated life situation of the living system (Bauer, 2008). While living within situations of stress, the genetic mechanisms simulate the outcome of modifications of the genetic code prior to actually doing the modification. Newborn species receive this information (updated genetic code), if the simulation process has terminated successfully.

Back to the prior example: the creation process of new symbols from within a cognitive perspective. Spontaneous, but "errorness" modifications may cause the system to decrease the capability of receiving further information $(T)$. From a semantic perspective, the system will stop learning. $A$ practical example comes out of the Attention-Deficit Hyperactivity Disorder (ADHD or AD/HD). Although holding a relatively high intelligence, people have difficulty processing information as quickly and accurately as others. The environment has a tremendous influence, if those persons may continue to learn. But it is possible nowadays to overcome this blocking situation, which is caused by misinterpreting the behavior of those people.

Now, coming back to Rechenbergs question, what is information? Information causes all causal processes within this world. Information 'feeds' spontaneous processes as well. Information is the structural potential of any system in order to become completed. How can the content of a message be measured? The content of a message $a$ is given by the transformation $T$ which is happening to the receiver $b$. Identical messages may have different meanings to receivers, because T depends on the receiver. What we call "meaning" is not just a passive declaration of certain attributes to an object. Any T permits physical forces which are attempting to maximize (to 'complete') the number of internal states (based on the $2^{\text {nd }}$ law of thermodynamics). The form of language has been analyzed by Noam Chomsky (Chomsky, 1957). He has shown that any language is based on verbs and nouns, which can be embedded into a recursive, nested structure. We see at a glance that the output vector $b$ of our transformation $b=\mathrm{T}(a)$ represents the verb. This verb is the kind of activity which gets associated to the incoming message $a$. The noun is represented by the incoming message $a .^{2}$ Each cultural/ personal form is defined by a set of

\footnotetext{
${ }^{2}$ The basic concept is that a primary function of language is not only to represent "knowledge", but to support the process of increasing the amount and value of internal states. I have called this notion of semantics 'inverse semantics' (Luhn, 1999).
} 
executable transformations. This provides a direct measure about the degree of adaptation or assimilation of the receiver $b$. Additionally the degree of assimilation over time has to be measured, in comparison to the kinds of incoming messages. These measures deliver the concept of Capurro's formula: "information = instantiation of form". Each message is embedded in another code, which corresponds to the style of a message (i.e. collaborative, reflexive, imperative). The mode of reaction depends on the level of accessible spontaneous resources at the receiver, and shows following structure:

- the message itself is part of a causal process and causes further activations;

- those activations exists of super positioning (via association) internal motifs and other associated memory structures;

- possible spontaneous creations of new interconnections are included as well; we may think about all those irregular forms of snowflakes: even if a predefined code is used, the actual detailed activation will always end up in a configuration which is (slightly) new; if the spontaneous resources of the system are still active (not blocked; remember the example of ADHD), then the system will propagate new interrelations;

- the final action results of a super positioning of those activation patterns (may include body movement and further reflexions).

If the internal spontaneous resources are not active any more, then the outcome will always be a deterministic reaction. That is, the causal process behaves like a mechanistic force: a given input will be without any resistance translated into a deterministic output behavior. If there is a certain level of spontaneous resources given, then the causal process will always show structures of resistance: new interconnec-tions might be drawn. This may include (from an energetic standpoint) self-decided additional activations in order to release further reflexions (what we call "thinking").

The instance which takes this decision is the "whole" of the mind field, which is causally embedded into an overall arrangement within the whole of its environment. The goal is always, in terms of the $2^{\text {nd }}$ law, to increase the amount of internal states, by increasing the entropy of the world. If we learn to prepare a new kind of nurture, then we will increase the entropy of the world by transforming highly ordered nurture (low entropy) into disordered output (high entropy). "Mental causation" gives a name to the ever completing process of creating individual physical laws - of persons. The universe does not hold explicitly the processes which are happening in anybody's mind - because of the completion theorem. And the capability of language finally enables a new phase space of potentials and possible structures. By developing his "logic in reality" (LIR), Joseph Brenner opens the field to a new, causal information theory (Brenner, 2008).

To summarize, our symbols and behavior schemes are holding a homomorphism to the structures of the world. The isomorphic kernel reflects fundamental information such as physical laws. The residual structures are completing the world and attempt to maximize the total amount of states of all systems. Such system states are equivalent to what we call behavior schemes, knowledge or in-formation. Any living specie is relying on this heteronomous deep structure, and is forced and forcing by physical evidence to maximize the possible states, the in-formation of the world. Nature is giving to us this information in a very direct, non-metaphoric message: throughout the vocabulary which is made by natural laws, and the message which corresponds to further autonomy and freedom (the completion theorem). We are enabled to create and to enter into the information society by ontological evidence. Such insight into this logical fundament of our world will enable us to reinvigorate ourselves and to overcome existing blocking situations. It is the aim of all people which are dealing with a unified theory of information to work towards a 'networked solution', which may transform by itself the cultural structures into a new state (Capurro, 2009).

\section{Fundamentals in Computing Science}

The goal of a new concept within computer science is to overcome the gap between syntax and semantics. From within an application level, computer scientists are developing concepts in order 
to support semantic data representation. Terms like ontology's of computing systems / informatics, object orientation, semantic web and semantic databases illustrate this attempt. But the common computing architecture does not support concepts of semantics.

Living species are able to retrieve meaningful data by applying the concept of semantic message decoding. An incoming auditory and/or visual message gets immediately transformed into a known category. We recognize an apple or a person's face immediately and without any further thinking process. The identity of the recognized category is given through the transformation rule, the code. For instance, such a rule may map 2-dimensional projections of objects to one single object category. This is the way how visual recognition is working; auditory recognitions functions in the same manner. It is important to see that no further algorithm gets executed in order to retrieve such data. That is, from within a physical perspective such a data retrieval task may consume very little energy. Theoretically it may even consume zero energy, because the incoming signals are triggering causal chains and are feeding those chains with their own energy. This process may also run very fast, theoretically with the speed of light. The creation and storage of such categories consumes an amount of energy (because matter gets structured), but the retrieval process is very effective in terms of energy and speed.

The mostly used computing architecture today has been introduced by John von Neumann. But this architecture doesn't support the semantic conception, which has been described in the preceding sentences. It supports an optimized usage of the limited system resources of a computer from within a perspective of the concept of a Turing machine. The von Neumann architecture permits to store and execute flexible programs in order to fulfill the required computing task. Such computing task is given by the implementation of specific programs. The so called CPU (Central Processing Unit) executed many interim steps in order to receive the desired output. All those interim steps (computer scientists are speaking of the clock rate of the CPU) have to be stored and newly used. This costs huge amounts of energy and time.

Let us look now in a deeper way to typical information retrieval tasks. Applications like those of Google spread all their data in huge computer farms. Google may operate nowadays hundreds of thousands of computing nodes. If you start a question to the Google search engine, many sophisticated algorithms are getting executed in order to find the required location of your query (data center) and to finally retrieve your data. During this process, thousands of computer nodes will dissipate huge amounts of energy, because each interim result of all those algorithms has to be stored. This process of storing a huge amount of interim data (where the user has no idea about) costs energy and time. Google has successfully developed very fast algorithms, which use on the other hand huge amounts of hardware. A simple calculation is showing that more than hundred millions of dollars are required in order to pay the fee for electrical power per year.

A new computing architecture may overcome such problems. This concept permits to retrieve any required data within one or very few steps (instead hundreds of thousands of steps). A concept of semantics will be introduced from the beginning: this concept is built of the identity of any data in terms of 'meaning' and 'storage' by using what we have called a code. Let us look at an example: a list of names and some additional information. Using our language, we want to store all kinds of words, which are made of letters. Let us use a code, which exists of 5 bit in order to decode a single letter. We may decode up to 36 letters, which includes all letters of the alphabet. The letter "A" might be represented by the bit-pattern "00001". The name "TURING" is given by following pattern:

"1010010101100100100101110 00111"

In a next step we may store any other kind of data at this address (i.e. first name, birth date, telephone, email-address etc.).

The decoded message is identical to its address within the computing device. We conceptualize an identity between the meaning of any message to the user and its address in the computer. This approach gives the name to this concept: $\mathrm{iBIT}=$ identity Based Information Transformation. A basic example of this concept has already been defined; it incorporates a set of reversible transformations, which do not require the usage of a CPU (Luhn et. al., 2010). 
Please note, that up to now all kinds of computing programs and architecture do not rely on a certain information concept (triadic information), but on a statistical approach. Database systems execute sophisticated algorithms in order to calculate the storage location for kinds of data. Even elaborated pattern recognition systems are still based on intensive statistical analysis of picture data, rather than on fundamental analysis of certain data layers. A prototype of an industrial pattern recognition system has already successfully been developed and tested in microelectronics production. The core of this concept is a non-probabilistic, non-CPU based system which automatically identifies so called semiconductor wafers. Such wafers are made of monocrystalline silicium substrates and show very intensive and challenging light reflection characteristics. They are looking like metallic mirrors and change their color and reflectivity during the production process quite intensively. This new iBIT architecture holds the structure of a quantum computer: many simultaneous input patterns (vectors) may overlay at the same time, and deliver synchronously required output data.

Connectionist systems and the theory of neural networks are also conceptualizing vectors, which transfer (or represent) messages. But all those approaches (including classical data storage and retrieval concepts) conceptualize the 'meaning' of any data by a statistical, probabilistic analysis of any kind of data.

Nevertheless, the physicist Richard Feynman gave in a ground breaking speech in 1985 some hints about new directions (Feynman, 1996). Feynman participated in the 'Connection Machine Project' at the MIT in Boston. He investigated in parallel processing, in reversible computing and he predicted modern Nano technology. He also saw, that nature holds the capability of computing. His example was a magnet. The magnetic force (as a physical law) causes a specific field. The structure of this field gets calculated by this physical law. But he stays in Shannon's information paradigm and the classical theory of semantics. The meaning of any information, which gets processed by a computer, is only given by the user. Feynman didn't conceptualize the similarity (the conceptual identity) between the information, which gets processed by nature, and the information which gets processed in human minds. We are relying on kinds of certain data, and there is no reason why computing systems can't do likewise.

Another outcome of this approach is the elimination of the halting problem for those applications, which may not be executed on von Neumann-like computers. The existence of solutions to any process in the physical world has been shown. This is not true for computer programs. Alan Turing has even shown that it is not possible to predict if a computer program will once stop. As a conclusion, each program has to be validated and tested separately and very intensively. It has to be tested on the target hardware and the test should include all kinds of interactions of the users. Programs are made by different people. Their successful execution depends deeply on major preconditions: does the operating system support all required functionality? Do other programs, which may be called during runtime, fulfill their job? Does the user know how to interact with the program? Is the hardware powerful enough in order to provide acceptable response times? Those questions give reason why computer science is nowadays changing to an engineering discipline.

Three research projects have been started in order to explore and develop the iBIT concept at Technical University Dresden, Germany. The scientific goal of this project is to deliver a conceptual and theoretical framework, which enables to design computing systems, which enable maximum performance and minimum energy consumption. Hundreds of millions of dollars may be potential savings in the future, while enabling new kinds of functionality. But the existing computing concepts have been developed continuously over the last decades. So the target is a principal validation of the new approach and a stepwise integration into existing frameworks of applications. The final goal is a physical-semantic concept of computing based on certain sets of data and structures, rather than expanding on the existing probabilistic approach.

\section{References}

Annila, A. \& Kuismanen, E. (2009). Natural Hierarchy Emerges from Energy DIspersal. BioSystems, 95, 227-233.

Bauer, J. (2008). Das Kooperative Gen. Abschied vom Darwinismus. Hamburg: Hoffmann und Campe. 
Bohm, D. (1990). A New Theory of the Relationship of Mind and Matter. Philosophical Psychology, 3(2), 271-286.

Brenner, J. E. (2008). Logic in Reality. Springer.

Capurro, R. (1978). Information. Ein Beitrag zu Etymologischen und Ideengeschichtlichen Begründung des Informationsbegriffs. München: Saur.

Capurro, R. (2009). Past, Present and Future of the Concept of Information. triple-C, 7(2), 125-141.

Capurro, $\mathrm{R}$ (2011). What is Angeletics? Retrieved from http://www.capurro.de/angeletics.html

Chomsky, N. (1957). Syntactic Structures. Den Haag: Mouton.

Churchland, P.S. \& Sejnowski, T.J. (1992): The Computational Brain. Cambridge: MIT Press.

Feynman, R. P. (1996). Feynman Lectures on Computation. London: Penguin Books.

Feynman, R. P., Leighton, R. B. \& Sands, M. (2005). The Feynman Lectures on Physics. Vol. 1- 3. Boston: Addison Wesley.

Floridi, L (2003). Semantic Conception of Information. Retrieved from http://plato.stanford.edu/

Graßmann, H. (2007). On the Mathematical Structure of Messages and Message Processing Systems. Isomorph Letters A: Physics of Information, 1.

Graßmann, H. (2008). Ahnung von der Materie. Physik für Alle. Köln: DuMont.

Haugland, J. (1997). Mind Design II: Philosophy, Psychology, and Artificial Intelligence: Philosophy, Psychology, Artificial Intelligence. Cambridge: MIT Press.

Hiley, B.J. \& Pylkkänen, P. (2005). Can Mind Affect Matter Via Active Information? Mind \& Matter, 3(2), 7-27.

Kant, I. (1790). Kritik der Urteilskraft. Translation by James Creed Meredith retrieved from http://ebooks.adelaide.edu.au/k/kant/immanuel/k16j/

Kohonen, T. (2001). Self-Organizing Maps. Berlin: Springer.

Luhn, G. (1999). Implizites Wissen und Technisches Handeln am Beispiel der Elektronikproduktion. Bamberg: Meisenbach.

Luhn, G., Harter, J. \& Kreupl, F. (2010). Information Storage and Retrieval System. United States Patent Application 20100057685.

Penrose, R. (1989). The Emperor's New Mind Concerning Computers, Minds, and the Laws of Physics. Oxford: Oxford University Press.

Rechenberg, P. (2003). Zum Informationsbegriff der Informationstheorie. Informatik Spektrum, 26(5), 317-326.

Shapiro, J.A. (2006). Genome Informatics: The Role of DNA in Cellular Computations. Biological Theory 1(3), 288-301.

Vollmer, G. (1985). Das Alte Gehirn und die Neuen Probleme. In G. Vollmer (Ed.), Was können wir wissen? Band 1. Die Natur der Erkenntnis. Stuttgart: Hirzel.

Weizsaecker, C. F. (1971/1980). The Unity of Nature. New York: Farrar Straus \& Giroux.

Wiener, N. (1948). Cybernetics or Control and Communication in the Animal and the Machine. Cambridge: MIT Press.

Wilholt, T. (2004). Zahl und Wirklichkeit. Paderborn: Mentis.

\section{About the Author}

Gerhard Luhn

Senior Principal and Project lead at Technical University Dresden, Gerhard earned his PhD in introducing anthropological concepts to engineering design and automation (Analysis of the implicit dimension of acting and knowing). He led numerous international projects and teams in Microelectronics. His current research centers on new fundaments within an ontology of information and computing science. 